\title{
ON A DISCRETENESS CONDITION OF THE SPECTRUM OF SCHRÖDINGER OPERATORS WITH UNBOUNDED POTENTIAL FROM BELOW' ${ }^{1}$
}

\author{
V. BENCI AND D. FORTUNATO
}

\begin{abstract}
We obtain a discreteness condition for the spectrum of the Schrödinger operator $-\Delta+V(x)$ in a case in which $V$ is not bounded from below.
\end{abstract}

0. Introduction. Discreteness conditions of the spectrum of the Schrödinger operators $H=-\Delta+V(x)$ ( $\Delta$ the Laplacian and $V$ a potential) have been studied by several authors under the assumption that $V$ is bounded from below (cf., e.g. A. M. Molchanov [5], M. Schechter [6], I. M. Glazman [4]). In a recent paper [1] we have proved that if $V \in L_{\text {loc }}^{2}\left(R^{n}\right), V(x)>0$ and $\int_{S\left(x_{0}\right)} V(x)^{-1} d x \rightarrow 0$ for $\left|x_{0}\right| \rightarrow+\infty\left(S\left(x_{0}\right)\right.$ is the unit sphere centered at $\left.x_{0}\right)$, then the spectrum $\sigma(h)$ of the selfadjoint realization $h$ of $H$ in $L^{2}\left(R^{n}\right)$ consists of a denumerable set of eigenvalues of finite multiplicity. The proof is based on a compact embedding theorem of M. Berger and M. Schechter [3] and on the use of a suitable class of weighted Sobolev spaces introduced by the authors [2]. In the present paper we generalize the result of [1] to a case in which $V$ is not bounded from below; precisely, we assume that $V=V_{1}+V_{2}$ where $V_{1}, V_{2}$ satisfy the following assumptions:

$$
\begin{gathered}
\exists k>0 \text { s.t. inf ess } V_{1}(x)>-k \text { and } \int_{S\left(x_{0}\right)} d x /\left(V_{1}(x)+k\right) \rightarrow 0 \\
\quad \text { for }\left|x_{0}\right| \rightarrow+\infty \\
V_{2} \in L^{n / 2}\left(R^{n}\right) \text { for } n \geqslant 2 .
\end{gathered}
$$

1. Some preliminaries. Let $\rho_{0}, \rho_{1}$ be two positive Lebesgue measurable functions on $\left.R^{n}, p \in\right] 1,+\infty\left[\right.$. We denote by $\Gamma^{1, p}\left(R^{n}, \rho_{0}, \rho_{1}\right)$ the space of distribution $u$ on $R^{n}$ such that $\left(\rho_{0}\right)^{1 / p} u \in L^{p}\left(R^{n}\right)$ and $\left(\rho_{1}\right)^{1 / p}|\operatorname{grad} u| \in$ $L^{p}\left(R^{n}\right)$ equipped with the norm

$$
\|u\|_{\Gamma^{1 \rho}\left(\mathbf{R}^{n}, \rho_{0}, \rho_{1}\right)}=\left(\int_{R^{n}} \rho_{0}(x)|u(x)|^{p} d x+\int_{R^{n}} \rho_{1}(x)|\operatorname{grad} u(x)|^{p} d x\right)^{1 / p} .
$$

Received by the editors January 24, 1977.

AMS (MOS) subject classifications (1970). Primary 35P05; Secondary 81A09.

Key words and phrases. Discrete spectrum, Schrödinger operator, Sobolev spaces, compact embedding.

'Results obtained at the Courant Institute of Mathematical Sciences, New York University, under the sponsorship of the Italian "Consiglio Nazionale delle Ricerche". 
$\dot{\Gamma}^{1, \rho}\left(R^{n}, \rho_{0}, \rho_{1}\right)$ is the closure of $C_{0}^{\infty}\left(R^{n}\right)$ in $\Gamma^{1, p}\left(R^{n}, \rho_{0}, \rho_{1}\right)$. As usual, we put $\Gamma^{1,2}\left(R^{n}, 1,1\right)=H^{1}\left(R^{n}\right)$. We shall denote by $S\left(x_{0}\right)$ the unit ball in $R^{n}$ centered at $x_{0} \in R^{n}$. Let us recall the following compact embedding theorem (cf. [1, Theorem 2.1]).

THEOREM 1.1. Let us suppose that inf ess $\rho_{0}(x)>0$, inf ess $\rho_{1}(x)>0$ and $\int_{S\left(x_{0}\right)} \rho_{0}(x)^{-1} d x \rightarrow 0$ for $\left|x_{0}\right| \rightarrow+\infty$. Then the embedding $\Gamma^{1, \rho}\left(R^{n}, \rho_{0}, \rho_{1}\right) \hookrightarrow$ $L^{p}\left(R^{n}\right)$ is compact.

2. The results. Let $V \in L_{\text {loc }}^{1}\left(R^{n}\right)$ be a real potential which admits the decomposition $V=V_{1}+V_{2}$, with $V_{1}, V_{2}$ measurable, and real functions satisfying the following assumptions:

(i) $\exists k>0$ such that inf ess $V_{1}(x)>-k$,

(ii) $\int_{S\left(x_{0}\right)}\left(V_{1}(x)+k\right)^{-1} d x+k \rightarrow 0$ for $\left|x_{0}\right| \rightarrow+\infty$,

(iii) $V_{2} \in L^{n / 2}\left(R^{n}\right)$ for $n \geqslant 2$.

Let us consider the Hamiltonian operator formally defined by

$$
H u=-\Delta u+V(x) u
$$

and the associated sesquilinear form

$$
a(u, v)=\int\left\{\sum_{i} u_{x_{i}}(x) \overline{v_{x_{i}}(x)}+V(x) u(x) \overline{v(x)}\right\} d x, \quad u, v \in C_{0}^{\infty}\left(R^{n}\right) .
$$

In the following we shall put $W=\stackrel{\circ}{\Gamma}^{1,2}\left(R^{n}, V_{1}+k, 1\right)$ and its scalar product will be denoted by $(\cdot \mid \cdot)_{W}$.

Proposition 2.1. The form $(a \cdot, \cdot)$ can be continuously extended to $W \times W$.

Proof. By virtue of (iii) and the Sobolev embedding theorem, we have $\forall_{v}^{u} \in C_{0}^{\infty}\left(R^{n}\right)$,

$$
\begin{aligned}
|a(u, v)| \leqslant & \left|(u \mid v)_{W}\right|+k\left|\int u(x) \overline{v(x)} d x\right|+\left|\int V_{2}(x) u(x) \overline{v(x)} d x\right| \\
\leqslant & \leqslant u\left\|_{W} \cdot\right\| v\left\|_{W}+k\right\| u\left\|_{L^{2}\left(R^{n}\right)} \cdot\right\| v \|_{L^{2}\left(R^{n}\right)} \\
& +\left\|V_{2}\right\|_{L^{n / 2}\left(R^{n}\right)} \cdot\|u\|_{L^{2 *}\left(R^{n}\right)} \cdot\|v\|_{L^{2 *}\left(R^{n}\right)} \\
\leqslant & c_{1}\|u\|_{W} \cdot\|v\|_{W}+c_{2}\left\|V_{2}\right\|_{L^{n / 2}\left(R^{n}\right)}\|u\|_{H^{\prime}\left(R^{n}\right)} \cdot\|v\|_{H^{1}\left(R^{n}\right)} \\
\leqslant & c_{3}\|u\|_{W} \cdot\|v\|_{W}
\end{aligned}
$$

where $2^{*}=2 n /(n-2)$ and $c_{1}, c_{2}, c_{3}$ are positive constants. Q.E.D.

TheOREM 2.2. Let (i), (ii), (iii) be satisfied. Then there exists a unique selfadjoint operator $h: D(h) \rightarrow L^{2}\left(R^{n}\right)$ so that $D(h) \subset W$ and $(h u \mid v)_{L^{2}\left(R^{n}\right)}=$ $a(u, v)$ for $u \in D(h), v \in W$. Moreover the spectrum $\sigma(h)$ of $h$ is formed by a sequence $\left\{\lambda_{k}\right\}$ bounded from below of isolated eigenvalues of finite multiplicity and

$$
L^{2}\left(R^{n}\right)=\sum_{k} M_{k}, \quad M_{k} \perp M_{k^{\prime}} \text { for } k \neq k^{\prime},
$$


where $M_{k}$ is the eigenmanifold corresponding to $\lambda_{k}$.

Let us initially prove the following

LEMMA 2.3. There exists $\lambda_{0}>0$ such that for each $\phi \in C_{0}^{\infty}\left(R^{n}\right)$ :

$$
\begin{aligned}
\alpha\|\phi\|_{W}^{2} & \geqslant \int\left\{|\operatorname{grad} \phi(x)|^{2}+V(x)|\phi(x)|^{2}+\lambda_{0}|\phi(x)|^{2}\right\} d x \\
& \geqslant \beta\|\phi\|_{W}^{2}
\end{aligned}
$$

where $\alpha, \beta$ are positive constants.

Proof. $V_{2} \in L^{n / 2}\left(R^{n}\right)$, therefore (cf. Lemma 3.1 of [8]) for each $\varepsilon>0$ there exist two functions $\theta, \eta$ such that $\theta \in L^{\infty}\left(R^{n}\right),\|\eta\|_{L^{n / 2}\left(R^{n}\right)}<\varepsilon$ and $V_{2}=\theta+\eta$; therefore we have, $\forall \phi \in C_{0}^{\infty}\left(R^{n}\right)$,

$$
\begin{aligned}
& \int\left\{|\operatorname{grad} \phi(x)|^{2}+V(x)|\phi(x)|^{2}\right\} d x \\
& \quad=\|\phi\|_{W}^{2}-k\|\phi\|_{L^{2}\left(R^{n}\right)}^{2}+\int(\eta(x)+\theta(x))|\phi(x)|^{2} d x .
\end{aligned}
$$

On the other hand, by virtue of the Sobolev embedding theorem, we have

$$
\begin{aligned}
\mid \int(\eta(x) & +\theta(x))|\phi(x)|^{2} d x \mid \\
& \leqslant\|\theta\|_{L^{\infty}\left(R^{n}\right)} \cdot\|\phi\|_{L^{2}\left(R^{n}\right)}^{2}+\|\eta\|_{L^{n / 2}\left(R^{n}\right)} \cdot\|\phi\|_{L^{2 *}\left(R^{n}\right)}^{2} \\
& \leqslant\|\theta\|_{L^{\infty}\left(R^{n}\right)} \cdot\|\phi\|_{L^{2}\left(R^{n}\right)}^{2}+c_{1} \varepsilon\|\phi\|_{H^{1}\left(R^{n}\right)}^{2} \\
& \leqslant\|\theta\|_{L^{\infty}\left(R^{n}\right)} \cdot\|\phi\|_{L^{2}\left(R^{n}\right)}^{2}+c_{2} \varepsilon\|\phi\|_{W}^{2}
\end{aligned}
$$

where $c_{1}, c_{2}$ are positive constants and $2^{*}=2 n /(n-2)$. From (2.1) and (2.2) the conclusion easily follows if we choose $\varepsilon \leqslant c_{2} / 2$ and $\lambda_{0} \geqslant k+\|\theta\|_{L^{\infty}\left(R^{n}\right)}$. Q.E.D.

Let us now prove Theorem 2.2. If ${ }_{v}^{u} \in C_{0}^{\infty}\left(R^{n}\right)$ we set

$$
a(u, v)+\lambda_{0}(u \mid v)_{L^{2}\left(R^{n}\right)}=[u, v] .
$$

By virtue of Lemma 2.3, $W$ is isomorphic to the Hilbert space $U$ completion of $C_{0}^{\infty}\left(R^{n}\right)$ with respect to the scalar product $[\cdot, \cdot]$; therefore, by (ii) and Theorem 1.1, the embeddings $i: U \hookrightarrow L^{2}\left(R^{n}\right)$ and its adjoint $i^{*}:\left(L^{2}\left(R^{n}\right)\right)^{\prime} \hookrightarrow$ $U^{\prime}$ are compact. Now the proof of the theorem follows from standard arguments (cf., e.g., the proof of Theorem 4.1 of [1] and Lemma II.6 of [7]). Q.E.D.

REMARK 2.4. We observe that (0.2) may be replaced by some other assumption which assures that the sesquilinear form $a(u, v)$ is bounded from below (cf., e.g., [6] and [7]). In fact, if $a(u, v)$ is bounded from below, Lemma 2.3 and, hence, Theorem 2.2 are still valid.

\section{REFERENCES}

1. V. Benci and D. Fortunato, Discreteness conditions of the spectrum of Schrödinger operators, J. Math. Anal. Appl. (to appear) 
2. (5) 14-B (1976), 832-843.

3. M. S. Berger and M. Schechter, Embedding theorems and quasi-linear elliptic boundary value problems for unbounded domains, Trans. Amer. Math. Soc. 172 (1972), 261-278.

4. I. M. Glazman, Direct methods of qualitative spectral analysis of singular differential operators, Israel Program for Scientific Translation, Jerusalem, 1965.

5. A. M. Molchanov, Ob usloviayakh discretinosti spekra samosopryazhennykh differentsial'nykh uravnenji vtorogo poryadka. (The conditions for the discreteness of the spectrum of selfadjoint second-order differential equations), Trudy Moskov. Mat. Obšc. 2 (1953), 169-200.

6. M. Schechter, Spectra of partial differential operators, North-Holland, Amsterdam, 1971.

7. B. Simon, Quantum mechanics for Hamiltonians defined as quadratic forms, Princeton, Univ. Press, Princeton, N. J., 1971.

8. G. Stampacchia, Le problème de Dirichlet pour les équations elliptiques du second ordre $\grave{a}$ coefficients discontinus, Ann. Inst. Fourier (Grenoble) 15 (1965), 189-258.

Courant Institute of Mathematical Sciences, New York University, 251 Mercer Street, New YoRK, New York 10012

Current address (V. Benci): Istituto di Matematiche Applicate "U. Dini", Università di Pisa, 56100 Pisa, Italy

Current address (D. Fortunato): Istituto di Matematica Applicata, Università di Bari, Bari 70100, Italy 